\title{
Medium-Sensitive Fluorophore as a Moisture Probe in Polymer Film
}

\author{
Soichi OTSUKI and Kimihiro ADACHI \\ Osaka National Research Institute, AIST, 1-8-31 Midorigaoka, Ikeda 563, Japan
}

(Received August 23, 1993)

\begin{abstract}
Some aminonaphthalenesulfonate derivatives were examined as moisture probes in hydroxypropyl cellulose film. The fluorescence maximum in wavenumber and maximum intensity correlate linearly with water content in the film. The dependence of these values on relative humidity and water content is interpreted in terms of polarity increase of the microenvironment in the film. The difference in photophysical behavior between the fluorophores and characteristics of the polymer as a vehicle are discussed.

KEY WORDS Moisture Probe / Polymer Film / Hydroxypropyl Cellulose / Aminonaphthalenesulfonate / Fluorescence / Relative Humidity /
\end{abstract}

Fluorescence is very useful to study the properties of systems containing macromolecules. ${ }^{1,2}$ The emission from a fluorophore, i.e., spectrum and intensity of the emitted radiation, frequently depends on the properties of the medium, i.e., its polarity and rigidity. Medium-sensitive fluorophores have been used to characterize the physicochemical properties of polymers in solution ${ }^{3-7}$ or as solids. ${ }^{8-11}$ The sorption and diffusion of gases and vapors into solid polymers are of considerable practical and theoretical importance. Recently changes in absorption spectrum of solvatochromic dyes such as Reichardt's betaine dye have been used to characterize the sorption properties of polymers for moisture $^{12}$ and organic solvent vapor. ${ }^{13}$ However, only a few reports have utilized the fluorescence technique to characterize solid polymers exposed to various gases and vapors. ${ }^{14}$

In a previous study ${ }^{15}$ 5-dimethylamino-1naphthalenesulfonic acid (DNSA) embedded in a hydroxypropyl cellulose (HPC) film was characterized under varying ambient relative humidity (RH) by fluorescence spectroscopy. In this study, the dependence of fluorescence properties on $\mathrm{RH}$ and content of adsorbed water was examined for other two fluorophores, i.e., 5-dimethylamino-1-naphthalenesulfonamide (DNSM) and 8-anilino-1-naphthalenesulfonic acid (ANSA) in HPC films. The results for DNSM and ANSA were compared with those for DNSA. The difference in photophysical behavior between the fluorophores is discussed. The characteristics of HPC as a vehicle were determined from the effect of fluorophore concentration on the fluorescence properties and from the sorption behavior of water vapor.

\section{EXPERIMENTAL}

\section{Film Preparation}

DNSA (99\%; Aldrich), DNSM (99\%; Aldrich), ANSA (97\%; Aldrich), and HPC (L type; molar substitution, 1.89; Nippon Soda) were used as received. Ethanol solutions were prepared with various concentrations of a dye and $3.8 \%$ (g per $100 \mathrm{ml}$ solution) of HPC. Films were prepared by casting the solution on $45 \times 12 \times 1.1 \mathrm{~mm}$ quartz plates and allowing the solvent to evaporate. The thickness of the 
resulting films was about $17 \mu \mathrm{m}$ as calculated from the volume of the cast solution and the density of dry HPC, $1.22 \mathrm{~g} \mathrm{ml}^{-1}$.

\section{Fluorescence Measurements}

The fluorescence spectrum and intensity of the films were measured under nitrogen streams with various RHs. Excitation at $330 \mathrm{~nm}$ for DNSA and DNSM or at $350 \mathrm{~nm}$ for ANSA was used together with excitation and emission slits of $3 \mathrm{~nm}$. A sharp-cut filter (L38; Hoya) was placed on the emission side to reduce secondary diffracted and stray light. The gas flow system was the same as in the previous paper. ${ }^{15}$ Measurements were carried out at $25.0 \pm 0.2^{\circ} \mathrm{C}$. Detail procedures can be found in the previous paper. ${ }^{15}$

\section{Sorption Measurements}

The samples used were films cast on a $18 \times 18 \mathrm{~mm}$ glass plate. Water vapor sorption was measured at $25.0 \pm 0.2^{\circ} \mathrm{C}$ with a Cahn 2000 electrobalance and a gas flow system. Detail procedures are in the preceding paper. ${ }^{16}$

\section{RESULTS AND DISCUSSION}

The fluorescence spectra of the fluorophores in HPC films at various RHs are shown in Figures 1(a)-(c). Similar changes in spectrum with RH can be seen for all fluorophores. When RH increases, the fluorescence maximum $\left(v_{\max }\right)$ shifts to a lower frequency, accompanied by increase in bandwidth. The maximum intensity $\left(I_{\max }\right)$ decreases.

Fluorescence energies, bandwidths, and quantum yields of the aminonaphthalenesulfonate (ANS) derivatives show marked dependence on the dielectric constant of the medium. ${ }^{17-19}$ Because the above spectral changes with increasing RH in HPC films are similar to those observed in solution when the solvent is changed from nonpolar to polar one, they are clearly due to increase of the microenvironmental polarity of the film. It is expected that the microenvironment of dry
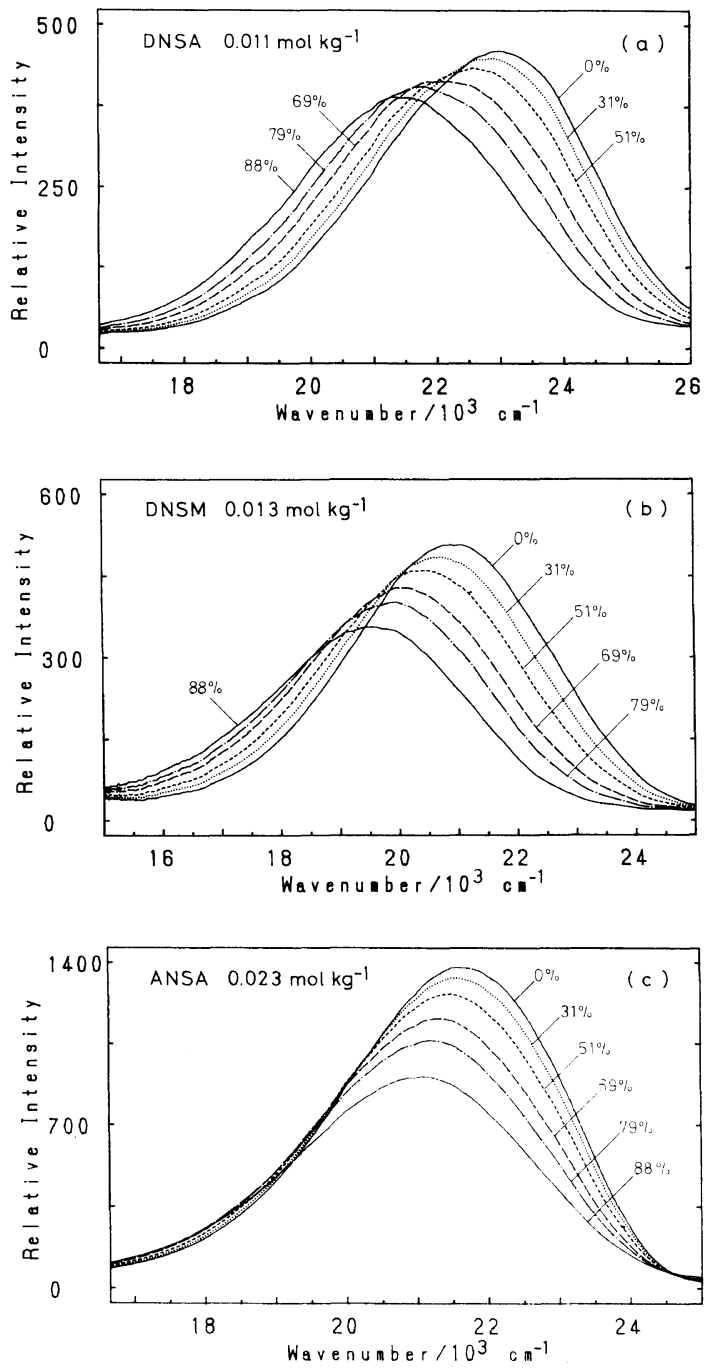

Figure 1. Effect of $\mathrm{RH}$ on the corrected fluorescence spectrum in an HPC film.

HPC is fairly nonpolar; when the film adsorbs water vapor, the polarity of this environment increases owing to the strong polar nature of water.

$v_{\max }$ and relative value of $I_{\max }$ for three different concentrations of the fluorophores are plotted as a function of $\mathrm{RH}$ in Figures 2(a)-(c). The dependence of these values on RH is nonlinear. The values vary slightly in the low RH region but vary much more in the high RH region. To clarify the difference 
(a)

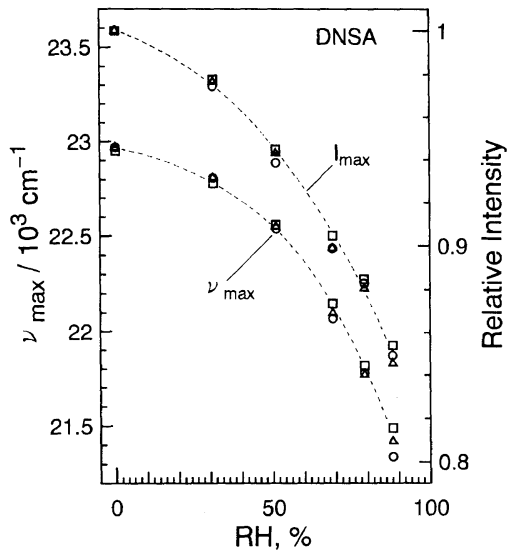

(b)

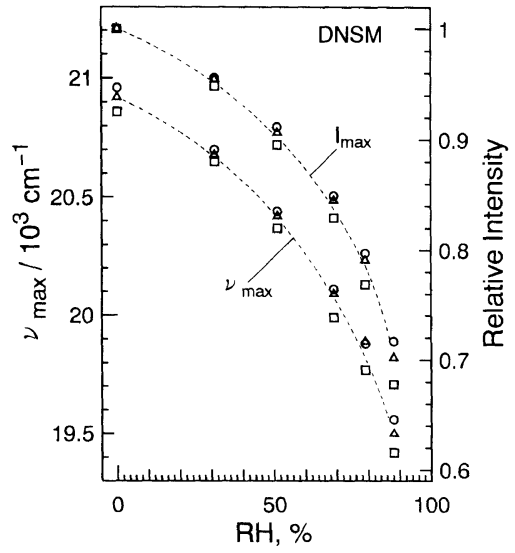

(c)

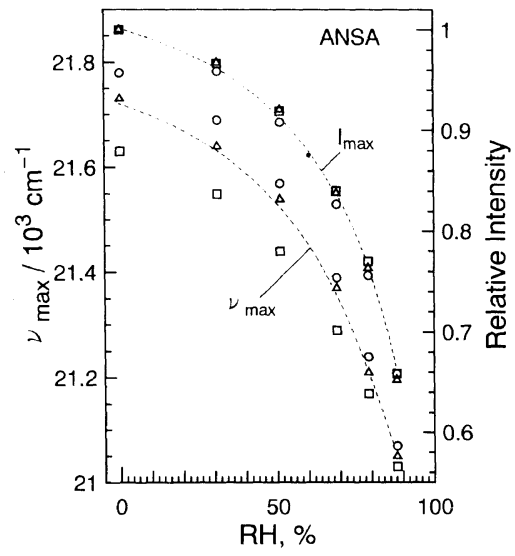

Figure 2. Relation of $v_{\max }$ and $I_{\max }$ to RH.

(a) DNSA Concentration: $0.0040 \mathrm{~mol} \mathrm{~kg}^{-1}$ (O); 0.011 mol kg ${ }^{-1}(\triangle) ; 0.022 \mathrm{~mol} \mathrm{~kg}^{-1}(\square)$.

(b) DNSM Concentration: $0.0067 \mathrm{~mol} \mathrm{~kg}^{-1}(\mathrm{O}) ; 0.013$ $\mathrm{mol} \mathrm{kg}^{-1}(\triangle) ; 0.027 \mathrm{~mol} \mathrm{~kg}^{-1}(\square)$.

(c) ANSA Concentration: $0.0058 \mathrm{~mol} \mathrm{~kg}^{-1}$ (O); 0.012 $\mathrm{mol} \mathrm{kg}{ }^{-1}(\triangle) ; 0.023 \mathrm{~mol} \mathrm{~kg}^{-1}(\square)$. between the three fluorophores, the sensitivities of $v_{\max }$ and $I_{\max }$ to $\mathrm{RH}$ changes are compared with one another. The differences in $v_{\max }$ between $0 \%$ and $88 \% \mathrm{RH}$ are $1460-1630$ $\mathrm{cm}^{-1}$ (DNSA), $1400-1440 \mathrm{~cm}^{-1}$ (DNSM), and $600-710 \mathrm{~cm}^{-1}$ (ANSA). The $I_{\max }$ decrements with increasing $\mathrm{RH}$ from $0 \%$ to $88 \%$ RH are $14.6 \%-15.5 \%$ (DNSA), $28.3 \%$ $32.2 \%$ (DNSM), and $34.1 \%-34.7 \%$ (ANSA). Although these values have some scatter as the concentration of fluorophore changes, it is concluded that the sensitivity of $v_{\max }$ to $\mathrm{RH}$ increases in the order of ANSA $<$ DNSM $<$ DNSA, whereas the sensitivity of $I_{\max }$ to $\mathrm{RH}$ increases in the order of DNSA $<$ DNS$\mathrm{M}<$ ANSA.

The fluorescence behavior of ANS derivatives has been reported to arise from two excited singlet states, the polar state having intramolecular charge-transfer (ICT) configuration and the nonpolar state. ${ }^{20,21}$ In a polar medium, the ICT state interacts more strongly than the nonpolar state. This interaction lowers the energy of the ICT state below that of the nonpolar state. The dependence of $v_{\max }$ on RH described above suggests that the relative contribution of the emission from the ICT state becomes greater in the order of $\mathrm{AN}$ $\mathrm{SA}<\mathrm{DNSM}<\mathrm{DNSA}$. It has been demonstrated that electron transfer from the excited molecule to the surrounding medium occurs as a fast nonradiative process from the excited state in an aqueous medium. ${ }^{22-25}$ The dependence of $I_{\max }$ on $\mathrm{RH}$ described above suggests that this process becomes more significant in the order of DNSA $<$ DNSM $<$ ANSA.

Figures 2(a)-(c) also show the effect of the fluorophore concentration on $v_{\max }$ and $I_{\max }$. Both $v_{\max }$ and relative value of $I_{\max }$ of DNSA are virtually independent of the fluorophore concentration. In the case of DNSM, however, $v_{\max }$ shifts to a lower frequency and the relative value of $I_{\max }$ decreases when a higher concentration of DNSM is used. In contrast, $v_{\max }$ of ANSA gets lower with increasing its 


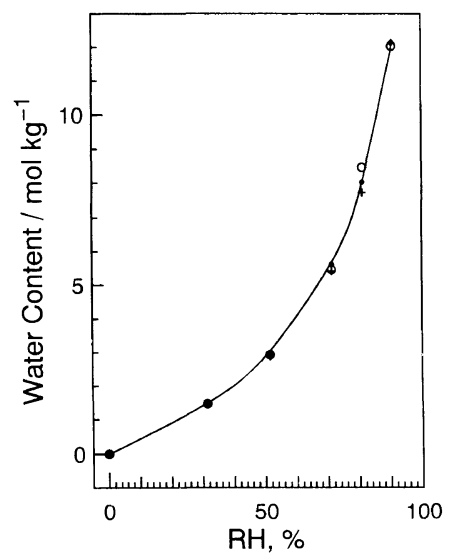

Figure 3. Relation of water content (mol per kg of dry film) to RH for HPC films with and without fluorophores; $0.022 \mathrm{~mol} \mathrm{~kg}^{-1}$ DNSA (•); $0.027 \mathrm{~mol} \mathrm{~kg}^{-1}$ DNSM (O); $0.023 \mathrm{~mol} \mathrm{~kg}^{-1}$ ANSA (+); none (-).

concentration particularly in the low $\mathrm{RH}$ range, whereas the concentration has little effect on the relative value of $I_{\max }$. These data are useful for using fluorophores as moisture probes.

The amount of adsorbed water vapor was measured at several RHs for HPC films with and without the fluorophores. In Figure 3, the water content increases with RH slowly in the low $\mathrm{RH}$ region and rapidly in the high $\mathrm{RH}$ region for all films. It can be seen that the fluorophores have little influence on the amount of water vapor adsorbed by HPC.

Figures 4(a)-(c) show plots of $v_{\max }$ and relative value of $I_{\max }$ against water content. The water content at each $\mathrm{RH}$ was determined from the isotherm of water vapor sorption shown in Figure 3. Linear regression was applied to the experimental points. The correlation coefficients of $v_{\max } v s$. water content are 0.993 (DNSA), 0.994 (DNSM), and 0.994 (ANSA). The correlation is fairly good for all fluorophores. The coefficients of $I_{\max } v s$. water content are 0.980 (DNSA), 0.998 (DNSM), and 0.998 (ANSA). The correlation of $I_{\max }$ is excellent for DNSM and ANSA. The linear dependence of $v_{\max }$ and $I_{\max }$ indicates that these values for the fluorophores can be used as good measures of the amounts of adsorbed water in
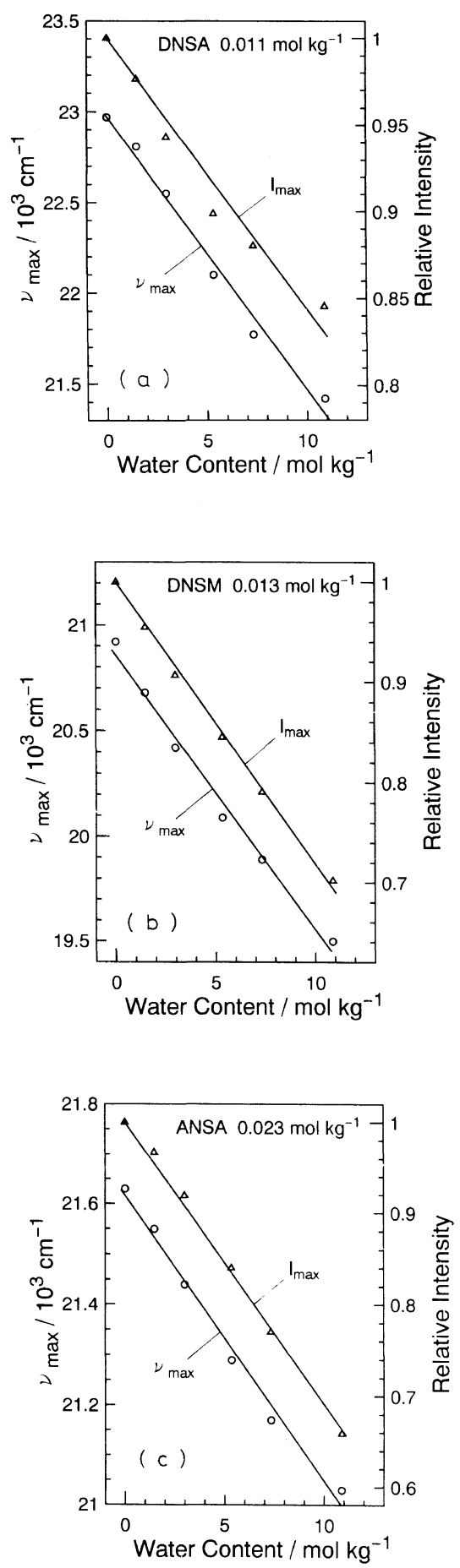

Figure 4. Relation of $v_{\max }$ and $I_{\max }$ to water content. The straight line should pass through the point $(0,1)$ for the relative value of $I_{\max }$. 
HPC film. To discuss the results theoretically, the fluorescence quantum yield should be used in stead of $I_{\max }$. The integrated area under the fluorescence curve is considered nearly proportional to the quantum yield. This value showed good linear dependence on water content in a similar manner to $I_{\max }$. Since $I_{\max }$ is more easily determined than the integrated area, $I_{\max }$ can be employed as an alternative to the fluorescence quantum yield of these fluorophores in HPC film.

Because the solvent dependence of the absorption maximum of ANS derivatives is very small, ${ }^{19-21}$ it is expected that the absorption maxima of the fluorophores used here are virtually unchanged in the HPC film even when the film fully adsorbs water vapor. The Stokes shift in wavenumber correlates linearly with the polarity parameter $\Delta f^{26}$ defined as

$$
\Delta f=(\varepsilon-1) /(2 \varepsilon+1)-\left(n^{2}-1\right) /\left(2 n^{2}+1\right)
$$

( $\varepsilon$, dielectric constant; $n$, refractive index)

Therefore, $v_{\max }$ should change linearly with $\Delta f$. Consequently, the linear dependence of $v_{\max }$ on water content probably arises from the fact that $\Delta f$ is nearly proportional to water content in the HPC film in the range of water content measured here. It seems that the convex nature of the dependence of $v_{\max }$ on $\mathrm{RH}$ reflects the concave nature of the isotherm of water vapor sorption. The decrease of $I_{\max }$ with increasing water content results from increase in the rate of the nonradiative process with increasing polarity of the medium. But the linear relationship between $I_{\max }$ and water content may be incidental.

Because the amount of water vapor adsorbed in the HPC film is not influenced by the presence of the fluorophores, the variation of $v_{\max }$ and $I_{\max }$ with fluorophore concentration shown in Figures 2(a)-(c) is not due to differences in sorption behavior. It is interesting that the variation of $v_{\max }$ and $I_{\max }$ with increasing fluorophore concentration is similar to that observed with increasing RH. HPC film exhibits some crystallinity. ${ }^{27}$ Dye molecules cannot enter the crystalline region. They do not distribute homogeneously throughout the entire amorphous region, but exist mainly within the region of higher flexibility. ${ }^{28}$ The ratio of fluorophore molecules which reside in the flexible region might increase when higher concentration is used. It has been demonstrated that water sorption of HPC follows the Flory model for lodlike mixing at a lower water content while it follows the Flory-Huggins model for random mixing at a higher water content. $^{29}$ It is expected that, when water content increases, disruption of the crystalline region progresses and the amorphous region increases. In addition, water molecules may mainly be adsorbed in the amorphous region having higher flexibility. Therefore, the average polarity of the microenvironment around fluorophore molecules probably increases when higher concentration of a fluorophore is used. This may become more significant at lower water content. This explains the effect of fluorophore concentration on $v_{\max }$ and $I_{\max }$ described above.

\section{REFERENCES}

1. G. Oster and Y. Nishijima, Fortschr. Hochpolym. Forsch., 3, 313 (1964).

2. M. A. Winnik, Ed., "Photophysical and Photochemical Tools in Polymer Science-Conformation, Dynamics, Morphology," Reidel, Dordrecht, 1986.

3. U. P. Strauss and G. Vesnaver, J. Phys. Chem., 79, 2426 (1975).

4. H. L. Chen and H. Morawetz, Eur. Polym. J., 19, 923. (1983).

5. Y. Wang and H. Morawetz, Macromolecules, 22, 154 (1989).

6. T. Seo, S. Take, K. Miwa, K. Hamada, and T. Iijima, Macromolecules, 24, 4255 (1991).

7. R. Hayashi, S. Tazuke, and C. W. Frank, Macromolecules, 20, 983 (1987).

8. R. O. Loutfy, Macromolecules, 16, 678 (1983).

9. K. J. Shea, D. Y. Sasaki, and G. J. Stoddard, Macromolecules, 22, 1722 (1989).

10. J. S. Royal and J. M. Torkelson, Macromolecules, 23, 3536 (1990)

11. L. W. Jenneskens, H. J. Verhey, H. J. van 
Ramesdonk, A. J. Witteveen, and J. W. Verhoeven, Macromolecules, 24, 4038 (1991).

12. R. A. McGill, M. S. Paley, and J. M. Harris, Macromolecules, 25, 3015 (1992).

13. M. H. Abraham, P. L. Grellier, R. A. McGill, R. M. Doherty, M. J. Kamlet, T. N. Hall, R. W. Taft, P. W. Carr, and W. J. Koros, Polymer, 28, 1363 (1987).

14. K. Y. Law, Polymer, 25, 399 (1984).

15. S. Otsuki and K. Adachi, J. Photochem. Photobiol. A: Chem., 71, 169 (1993).

16. S. Otsuki and K. Adachi, J. Appl. Polym. Sci., 48, 1557 (1993).

17. W. O. McClure and G. M. Edelman, Biochemistry, 5, 1908 (1966).

18. D. C. Turner and L. Brand, Biochemistry, 7, 3381 (1968).

19. C. J. Seliskar and L. Brand, J. Am. Chem. Soc., 93, 5414 (1971).

20. E. M. Kosower, H. Dodiuk, K. Tanizawa, M. Ottolenghi, and N. Orbach, J. Am. Chem. Soc., 97,
2167 (1975).

21. Y. H. Li, L. M. Chan, L. Tyer, R. T. Moody, C. M. Himel, and D. M. Hercules, J. Am. Chem. Soc., 97, 3118 (1975).

22. G. R. Fleming, G. Porter, R. J. Robbins, and J. A. Synowiec, Chem. Phys. Lett., 52, 228 (1977).

23. H. Nakamura and J. Tanaka, Chem. Phys. Lett., 78, 57 (1981).

24. G. W. Robinson, R. J. Robbins, G. R. Fleming, J. M. Morris, A. E. W. Knight, and R. J. S. Morrison, J. Am. Chem. Soc., 100, 7145 (1978).

25. R. A. Moore, J. Lee, and G. W. Robinson, J. Phys. Chem., 89, 3648 (1985).

26. N. Mataga, Y. Kaifu, and M. Koizumi, Bull. Chem. Soc. Jpn, 29, 465 (1956).

27. R. J. Samuels, J. Polym. Sci., A 2, 7, 1197 (1969).

28. S. Okajima, K. Nakayama, K. Kayama, and Y. Kato, J. Appl. Polym. Sci., 14, 1069 (1970).

29. J. S. Aspler and D. G. Gray, Macromolecules, 12, 562 (1979). 